\title{
Fully quantitative major and trace element laser ablation ICP mapping of heterogeneous samples
}

\author{
EMMA L TOMLINSON ${ }^{1}$, THOMAS RIEGLER ${ }^{1}$, JOSEPH A \\ PETRUS $^{2}$, SEAN H MCCLENAGHAN ${ }^{1}$ AND DAVID M \\ CHEW $^{1}$ \\ ${ }^{1}$ Trinity College Dublin \\ ${ }^{2}$ Laurentian University
}

Presenting Author: tomlinse@tcd.ie

Laser ablation inductively coupled plasma mass spectrometry (LA-ICP-MS) mapping provides a wealth of information on the distribution and mobility of trace elements in a range of igneous, metamorphic, sedimentary and ore minerals. Using modern instrumentation, two-dimensional trace element maps can be built up from a series of parallel line scans allowing for precise, ppm level analysis of elements with a spatial resolution of $<10 \mu \mathrm{m}$.

Quantitative laser ablation analysis utilizes an internal standard, either an element or combination of elements whose concentration is known and is used to anchor the absolute concentrations of all other elements in an analyzed mineral. Fully quantitative trace element maps can thus be produced for stoichiometric minerals and homogenous minerals when the internal standard concentration is known. However in the majority of samples, the internal standard concentration is variable as a result of zoning, inclusions, exsolution or alteration and therefore trace element maps for these samples are semiquantitative.

We present a method for producing fully quantitative trace element maps via either simultaneous split stream LA-ICPMS/OES mapping or by sequential major and trace element mapping of the same area by either LA-ICP-MS/OES or LAICP-MS. Absolute concentrations of major elements are quantified by normalization and the internal standard is then exported to the trace element dataset. The importing, synchronization and processing of data are implemented as plugins for Iolite 4. The combined major and trace element datasets can then be interrogated using Iolite add-on tools such as 'Monocle' (Petrus et al. 2017). This approach negates the need for spatial referencing of separate major and trace element maps and ensures that all data is collected at the same spatial resolution.

We discuss the experimental set-up required for synchronising simultaneous OES data collection with the sequential MS duty cycle and demonstrate the accuracy of this method using stoichiometric and homogenous minerals. We then illustrate the scope of the method for generating fully quantitative, spatially resolved trace element data for a diverse range of sample matrices including zoned igneous plagioclase, zoned metamorphic garnet and inter-grown and disseminated sulphides.

Petrus JA, Chew DM, Leybourne MI, Kamber BS (2017) Chemical Geology, 463, pp76-93. 